\title{
Erratum to: $A C E$ deletion polymorphism is associated with a high risk of non-infectious pulmonary complications after stem cell transplantation
}

Mitsuki Miyamoto $\cdot$ Makoto Onizuka $\cdot$ Shinichiro Machida $\cdot$ Masako Toyosaki Jun Amaki · Yasuyuki Aoyama · Hidetsugu Kawai · Ai Sato • Naoki Hayama • Yoshiaki Ogawa $\cdot$ Hiroshi Kawada $\cdot$ Kiyoshi Ando

Published online: 14 October 2014

(C) The Japanese Society of Hematology 2014

Erratum to: Int J Hematol (2014) 99:175-183

DOI 10.1007/s12185-013-1494-6

Unfortunately, Figs. 2 and 3 were swapped in the original publication of the article. The figures should appear as below.

The online version of the original article can be found under doi:10.1007/s12185-013-1494-6.

M. Miyamoto $\cdot$ M. Onizuka $(\bowtie) \cdot S$. Machida $\cdot$ M. Toyosaki

J. Amaki · Y. Aoyama · H. Kawai · A. Sato · Y. Ogawa ·

H. Kawada $\cdot$ K. Ando

Department of Hematology and Oncology, Tokai University

School of Medicine, 143 shimokasuya, Isehara,

Kanagawa 259-1143, Japan

e-mail:moni5@mac.com

N. Hayama

Department of Pulmonary Medicine, Tokai University School

of Medicine, Tokyo, Japan 


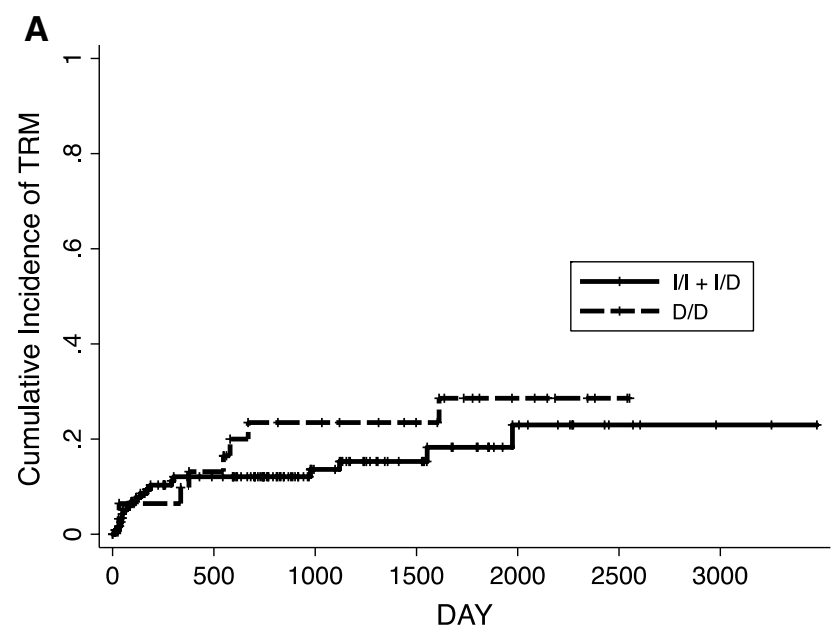

Fig. 2 Association of $A C E$ polymorphism to clinical outcomes. a Cumulative incidence curve of transplantation-related mortality (TRM) among allogeneic HSCT patients comparing the $A C E$ insertion genotypes and deletion polymorphisms (HR 1.53, $P=0.32$,

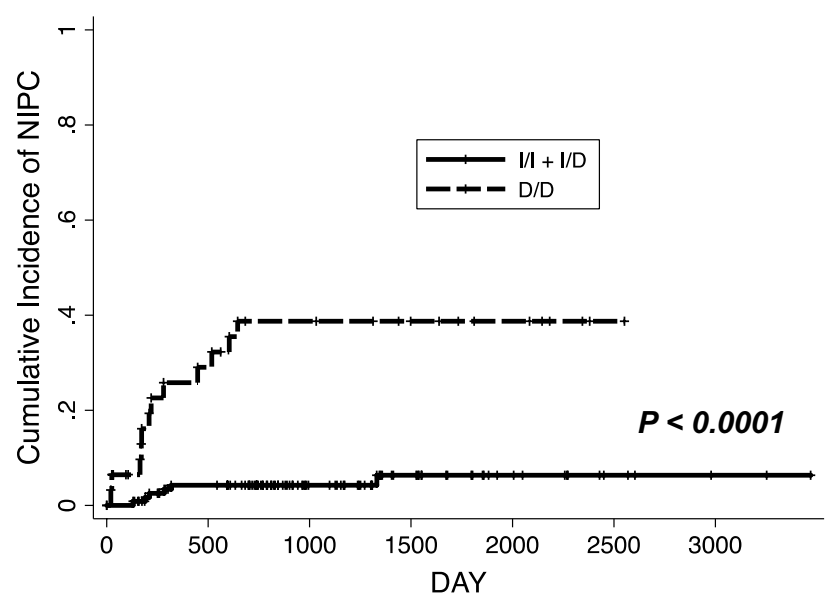

Fig. 3 NIPC and $A C E$ polymorphisms. Cumulative incidence curve of NIPC among allogeneic HSCT patients comparing the ACE insertion genotypes and deletion polymorphisms (HR 9.03, $P<0.0001$, $95 \%$ CI 3.4-24.2)

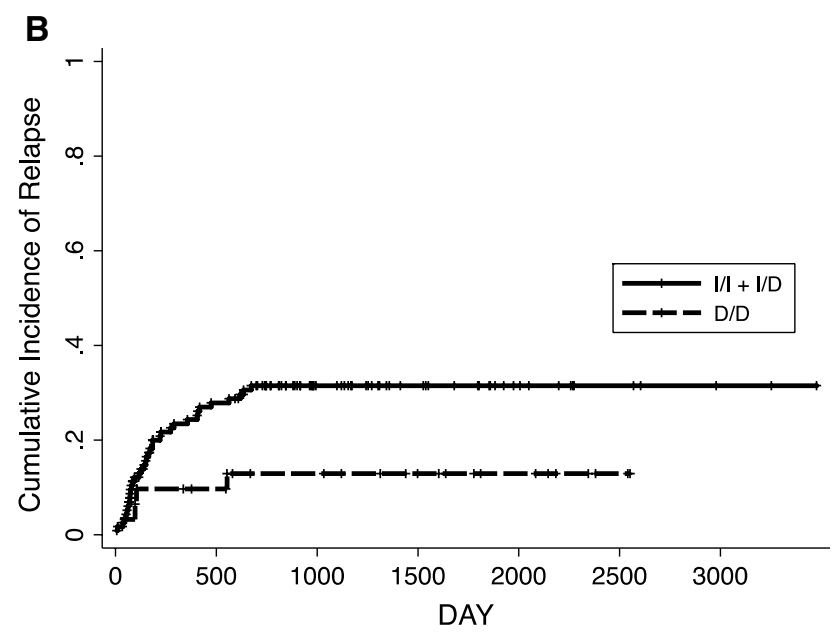

$95 \%$ CI 0.7-3.5). b Cumulative incidence curve of relapse among 140 allogeneic HSCT patients diagnosed with malignant disease comparing the $A C E$ insertion genotypes and deletion polymorphisms (HR $0.4, P=0.085,95 \%$ CI $0.1-1.1$ ) 